\title{
Demethylation of the HACE1 gene promoter inhibits the proliferation of human liver cancer cells
}

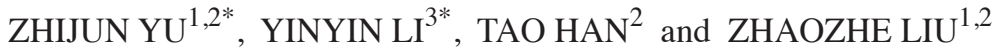 \\ ${ }^{1}$ Department of 2016 Levels of Integrated Traditional Chinese and Western Medicine Clinical Oncology, Liaoning \\ University of Traditional Chinese Medicine Graduate School, Shenyang, Liaoning 110847; ${ }^{2}$ Oncology Diagnosis \\ and Treatment Center, General Hospital of Shenyang Military Area Command, Shenyang, Liaoning 110016; \\ ${ }^{3}$ Department of Oncology, Shenyang Tenth People's Hospital, Shenyang, Liaoning 110849, P.R. China
}

Received August 7, 2018; Accepted February 6, 2019

DOI: $10.3892 / \mathrm{ol} .2019 .10139$

\begin{abstract}
HECT domain and ankyrin repeat containing E3 ubiquitin protein ligase 1 (HACE1) is frequently downregulated or lost in numerous types of cancer, including liver cancer. The aim of the present study was to examine whether demethylation of the HACE1 gene could inhibit tumour progression. The expression of HACE1 was detected in liver cancer cell lines. Clustered regularly interspaced short palindromic repeats (CRISPR)-CRISPR-associated (Cas)-based demethylation single guide RNAs for the HACE1 gene promoter were designed and transfected into liver cancer cells. Subsequently, proliferation was detected by MTT and colony formation assays, and optineurin (OPTN) ubiquitination and microtubule-associated proteins $1 \mathrm{~A} / 1 \mathrm{~B}$ light chain $3 \mathrm{~B}$ protein levels were detected by immunoblotting. The levels of HACE1 were significantly reduced in liver cancer cell lines compared with in a normal liver cell line. Demethylation of the HACE1 gene promoter increased HACE1 expression, inhibited the proliferation of liver cancer cells, and promoted OPTN ubiquitination and autophagy activity in liver cancer cells. In conclusion, activation of HACE1 expression by promoter demethylation may provide a suitable approach for anticancer therapy.
\end{abstract}

\section{Introduction}

Liver cancer has the fourth highest cancer mortality rate worldwide, and China is a country with a high incidence rate of liver cancer, where it accounts for $\sim 40 \%$ of the total number

Correspondence to: Dr Zhaozhe Liu, Oncology Diagnosis and Treatment Center, General Hospital of Shenyang Military Area Command, 83 Wenhua Road, Shenhe, Shenyang, Liaoning 110016, P.R. China

E-mail: zhijunzhaozhe@163.com

${ }^{*}$ Contributed equally

Key words: liver cancer cells, HECT domain and ankyrin repeat containing E3 ubiquitin protein ligase 1, demethylation, proliferation of cancer cases and cases of cancer-associated mortality $(1,2)$. Surgery remains the most effective treatment option for patients with liver cancer $(3,4)$. The majority of liver cancer cases are diagnosed at an advanced or unresectable stage; therefore, novel therapeutic strategies and therapeutic targets are required. Current studies mainly focus on the pathogenic genes and molecular mechanisms involved in liver cancer $(5,6)$.

HECT domain and ankyrin repeat containing E3 ubiquitin protein ligase 1 (HACE1) is a member of the HECT domain-containing E3 ligase family and was originally identified to be associated with the occurrence of Wilms' tumour (7). Furthermore, additional studies revealed that lower expression or mutations of HACE1 are associated with numerous types of human malignant tumours, including breast cancer, colorectal cancer, lung cancer, liver cancer, gastric cancer and lymphoma (5,8-12), which suggests that HACE1 functions as a tumour suppressor. Additional research concerning HACE1 has focused on a variety of downstream pathways; for example, HACE1 has been reported to act as a tumour suppressor by ubiquitinating optineurin (OPTN) and activating selective autophagy (10).

In mammalian cells, DNA methylation is critical for the regulation of gene expression and, therefore, serves a pivotal role in numerous physiological and pathological processes (13). DNA hypermethylation of tumour suppressor genes silences their expression and contributes to several types of human cancer $(9,14)$. Targeted DNA demethylation via the widely used clustered regularly interspaced short palindromic repeat (CRISPR)-CRISPR-associated (Cas) system has been widely reported on in recent years (15-17). A strategy for targeted demethylation of specific genomic loci by tethering Tet1-CD to the MS2 RNA element-containing single guide RNA (sgRNA) 2.0 system-guided dCas 9 and MS2 bacteriophage coat proteins was the first sgRNA and Cas9-mediated demethylation system to be reported $(15,17)$.

In the present study, low HACE1 expression was identified in human liver cancer cell lines compared with in a normal liver cell line. Subsequently, HACE1 expression was activated via targeted DNA demethylation using a two-plasmid system. Finally, in the present study, increased HACE1 expression was revealed to inhibit proliferation and activate selective autophagy in liver cancer cells. 


\section{Materials and methods}

Plasmid construction. The pdCas9-Tet1-CD and pcDNA3.1-MS2-Tet1-CD plasmids were provided by Professor Ronggui Hu (Chinese Academy of Sciences, Shanghai, China). sgRNAs targeting HACE1 were designed using an online tool (version 1.2; http://crispr.mit.edu/) as previously described (18). The designed sgRNAs (Table I) were synthesized as oligonucleotides (Sangon Biotech Co., Ltd., Shanghai, China), annealed and inserted into the pdCas9-Tet1-CD vector, which was digested with BbsI.

Cell culture and transfection. The human liver cancer cell lines HepG2 and Huh7, and the normal liver cell line L02 were obtained from the Cell Bank of the Chinese Academy of Sciences (Shanghai, China). The human liver cancer cell line Hep3B was purchased from the American Type Culture Collection (Manassas, VA, USA). All four cell lines have been authenticated by short tandem repeat profiling within the last 2 years. All cell lines were cultured in Dulbecco's modified Eagle's medium supplemented with $10 \%$ foetal bovine serum, $100 \mathrm{U} / \mathrm{ml}$ penicillin and $100 \mathrm{mg} / \mathrm{ml}$ streptomycin (all Gibco; Thermo Fisher Scientific, Inc., Waltham, MA, USA) in a $37^{\circ} \mathrm{C}$ humidified atmosphere with $5 \% \mathrm{CO}_{2}$. For the demethylation experiments, Hep3B or HepG2 cells $\left(10^{4}\right.$ cells/well $)$ were seeded into plates and the pdCas9-Tet1-CD $(10 \mu \mathrm{g})$ and pcDNA3.1-MS2-Tet1-CD $(8 \mu \mathrm{g})$ plasmids were transfected into cells using Lipofectamine ${ }^{\circledR} 2000$ (Thermo Fisher Scientific, Inc.). The subsequent experiments were performed $48 \mathrm{~h}$ post-transfection. For colony formation assays, cell lines with stable expression of the demethylation system were established as previously described (15).

Reverse transcription-quantitative polymerase chain reaction $(R T-q P C R)$. Total RNA was extracted from cells using a total RNA kit (Tiangen Biotech Co., Ltd., Beijing, China). cDNA was synthesized using the ReverTra Ace qPCR RT Master Mix (Toyobo Life Science, Osaka, Japan). The temperature protocol was as follows: Incubation at $37^{\circ} \mathrm{C}$ for $15 \mathrm{~min}, 50^{\circ} \mathrm{C}$ for $5 \mathrm{~min}$, and then $98^{\circ} \mathrm{C}$ for $5 \mathrm{~min}$. qPCR was performed to assess the relative abundance of HACE1 mRNA using specific primers (Table II) and SYBR Green dye (Toyobo Life Science) on an ABI 7500 fast real-time PCR system (Applied Biosystems; Thermo Fisher Scientific, Inc.). Thermocycling conditions were as follows: Initial denaturing at $94^{\circ} \mathrm{C}$ for $2 \mathrm{~min}$ followed by 40 cycles of $95^{\circ} \mathrm{C}$ for $15 \mathrm{sec}, 58^{\circ} \mathrm{C}$ for $15 \mathrm{sec}$ and $72^{\circ} \mathrm{C}$ for $30 \mathrm{sec}$. The relative abundance of HACE1 was normalized to that of GAPDH using the $2^{-\triangle \Delta C q}$ method (19). All data were obtained from at least three independent experiments.

Bisulphite DNA sequencing. Genomic DNA (gDNA) was extracted from cells of the indicated groups (control cells and cells transfected with sgRNA 1, 4 and 5) using the standard phenol-chloroform extraction method (20). gDNA was treated with bisulphite using the CpGenome Turbo Bisulphite Modification kit (EMD Millipore, Billerica, MA, USA) according to the manufacturer's protocol. The modified DNA was amplified using Platinum Taq DNA Polymerase (Thermo Fisher Scientific, Inc.) with the respective primer sets that recognize bisulphite-modified DNA only (Table II).
Table I. Sequences of the sgRNA target sites used in construction of the pdCas9-Tet1-CD expression plasmids.

\begin{tabular}{lc}
$\begin{array}{l}\text { sgRNA for } \\
\text { HACE1 }\end{array}$ & $\begin{array}{c}\text { Target site sequence } \\
\text { (protospacer adjacent motif region) }\end{array}$ \\
\hline sgRNA1 & 5'-GCCCTGGGCGGAGTCACGTTGGG-3' \\
sgRNA2 & 5'-GCGCCCAGGCCACGCCAACGCGG-3' \\
sgRNA3 & 5'-TGGGCGTACTCCTAAGCTTCTGG-3' \\
sgRNA4 & 5'-GAGTACGCCCAGTCGCTGCGTGG-3' \\
sgRNA5 & 5'-CCTGCCGGGCGGCTTTATGAGGG-3' \\
sgRNA6 & 5'-CCCTCATAAAGCCGCCCGGCAGG-3' \\
sgRNA7 & 5'-CGTTGATGATGTATGTTGGCTGG-3' \\
\hline
\end{tabular}

HACE1, HECT domain and ankyrin repeat containing E3 ubiquitin protein ligase 1; $\operatorname{sgRNA}$, single guide RNA.

The cycling parameters were as follows: Initial denaturing at $94^{\circ} \mathrm{C}$ for $2 \mathrm{~min}$, followed by 33 cycles of $95^{\circ} \mathrm{C}$ for $15 \mathrm{sec}, 58^{\circ} \mathrm{C}$ for $15 \mathrm{sec}$ and $72^{\circ} \mathrm{C}$ for $30 \mathrm{sec}$. Subsequently, the PCR products were cloned into the pMD18-T vector (Takara Bio, Inc., Otsu, Japan) and were sent for Sanger sequencing by Biosune Biotechnology, Co. (Shanghai, China).

Cell proliferation assay. Cells stably transfected with the demethylation system were seeded into a 96 -well plate (3,000 cells/well). For this experiment, $6 \mathrm{~h}$ post-cell seeding was defined as the $0 \mathrm{~h}$ time point. After 0,24 or $48 \mathrm{~h}$, the cells were incubated with MTT solution (cat. no. C0009; Beyotime Institute of Biotechnology, Haimen, China) for $4 \mathrm{~h}$ at $37^{\circ} \mathrm{C}$. The formazan product was then dissolved in dimethyl sulfoxide and quantified spectrophotometrically at a wavelength of $570 \mathrm{~nm}$ using a microplate reader (Bio-Rad Laboratories, Inc., Hercules, CA, USA). Experiments were performed in triplicate and repeated three times.

Colony formation assay. Cells stably transfected with the demethylation system were seeded into 6-well plates (1,000 cells/well). After 7 days, the cells were fixed with $4 \%$ paraformaldehyde (Sigma-Aldrich; Merck KGaA, Darmstadt, Germany) on ice for $30 \mathrm{~min}$ and stained with $0.1 \%$ crystal violet (cat. no. C0121; Beyotime Institute of Biotechnology) for $20 \mathrm{~min}$ at room temperature.

IP and immunoblotting. For IP, $\sim 6$ million cells transfected with the demethylation system were lysed in IP buffer (50 mM Tris-HCl, 5 mM EDTA, 0.1\% SDS and 1\% Nonidet P-40) supplemented with a protease inhibitor cocktail (Roche Diagnostics, Basel, Switzerland). Protein concentration of cell lysates was determined using the Pierce ${ }^{\mathrm{TM}}$ Bicinchoninic Acid Protein Assay Kit (Thermo Fisher Scientific, Inc.) and $2 \mathrm{mg}$ protein of the whole-cell-lysate was incubated with OPTN antibody (1:100 dilution; cat. no. 10837-1-AP; Proteintech Group, Inc., Chicago, IL, USA) and protein G agarose beads (cat. no. 16-266, Merck $\mathrm{KGaA}$ ) overnight at $4^{\circ} \mathrm{C}$. The immunoprecipitants were enriched and denatured at $100^{\circ} \mathrm{C}$ for $10 \mathrm{~min}$ in $2 \mathrm{X}$ SDS-PAGE loading buffer. The whole-cell-lysate input $(20 \mu \mathrm{g})$, and 
Table II. Sequences of the primers used in RT-qPCR and bisulphite DNA sequencing.

$\begin{array}{lll}\text { Target gene } & \text { Forward primer }\left(5^{\prime}-3^{\prime}\right) & \text { Reverse primer }\left(5^{\prime}-3^{\prime}\right)\end{array}$

Primers for RT-qPCR
GAPDH
HACE1

Primers for bisulphite

DNA sequencing

HACE1 promoter
ATTTGCCATGGGTGGAATCATATTG
CATCCTCAACATCAACATCACTGAC GCAAGAAATGGGCAGAAGAAATGTA

HACE1, HECT domain and ankyrin repeat containing E3 ubiquitin protein ligase 1; RT-qPCR, reverse transcription-quantitative polymerase chain reaction.

immunoprecipitants were then separated by SDS-PAGE (4\% concentrating gel and $10 \%$ separating gel), and transferred onto polyvinylidene difluoride membranes (Bio-Rad Laboratories, Inc.). Membranes were blocked in 5\% milk in TBS with $0.1 \%$ Tween-20 for $1 \mathrm{~h}$ at room temperature. The membranes were incubated at room temperature for $2 \mathrm{~h}$ with primary antibodies against ubiquitin (1:1,000 dilution; cat. no. sc-47721; Santa Cruz Biotechnology, Inc., Dallas, TX, USA), OPTN (1:2,000 dilution), HACE1 (1:1,000 dilution; cat. no. ab133637; Abcam, Cambridge, UK), GAPDH (1:5,000 dilution; cat. no. 60004-1-Ig; Proteintech Group, Inc.) and microtubule-associated proteins $1 \mathrm{~A} / 1 \mathrm{~B}$ light chain 3B (LC3; 1:500 dilution; cat. no. L7543; Sigma-Aldrich; Merck KGaA). Membranes were incubated with horseradish peroxidase(HRP)-conjugated goat anti-mouse IgG $(\mathrm{H}+\mathrm{L})$ or HRP-conjugated goat anti-rabbit $\operatorname{IgG}(\mathrm{H}+\mathrm{L})$ secondary antibodies (1:5,000 dilution; cat. nos. G-21040 and 31460; Thermo Fisher Scientific, Inc.) for $1 \mathrm{~h}$ at room temperature. Protein was then labelled by adding $1 \mathrm{ml}$ SuperSignal ${ }^{\mathrm{TM}}$ West Pico PLUS Chemiluminescent Substrate (cat. no. 34577; Thermo Fisher Scientific, Inc.). The signal was visualized using the Tanon 5200 Imaging System (Tanon Science and Technology Co., Ltd., Shanghai, China).

Statistical analysis. All experiments were performed in triplicate. All values are presented as the means \pm standard deviation. One-way analysis of variance was performed with Tukey's post hoc multiple comparisons test using GraphPad Prism software (version 5; GraphPad Software, Inc., La Jolla, CA, USA). $\mathrm{P}<0.05$ was considered to indicate a statistically significant difference.

\section{Results}

Lower expression of HACE1 in human liver cancer cell lines. To explore the expression profile of HACE1 in human liver cancer cell lines, lysates from three liver cancer cell lines (Hep3B, HepG2 and Huh-7) and one normal liver cell line (L-02) were prepared. Immunoblot analysis indicated that the protein levels of HACE1 decreased in the three liver cancer cell lines compared with in the normal liver cell line (Fig. 1A). The mRNA expression levels of HACE1 in the normal liver cell line were $\sim 4$ times higher than those in the liver cancer cell lines (Fig. 1B).
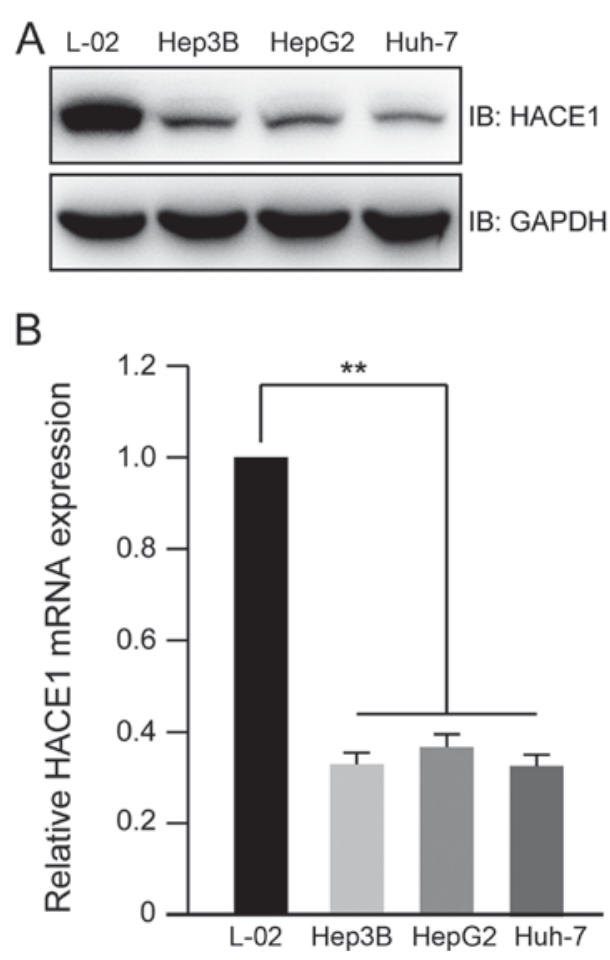

Figure 1. Expression profile of HACE1 in normal liver and liver cancer cell lines. (A) Protein expression profile of HACE1 in normal liver and liver cancer cell lines detected by immunoblotting. L-02 is a normal liver cell line; Hep3B, HepG2 and Huh-7 are liver cancer cell lines. (B) mRNA expression levels of HACE1 in normal liver and liver cancer cell lines detected by reverse transcription-quantitative polymerase chain reaction. Data are presented as the means \pm standard deviation of three independent experiments and were analysed using one-way analysis of variance with Tukey's post hoc test. ${ }^{* *} \mathrm{P}<0.01$. HACE1, HECT domain and ankyrin repeat containing E3 ubiquitin protein ligase 1 ; IB, immunoblotting.

CRISPR-Cas-based HACE1 promoter demethylation sgRNA design and activity detection. Using the previously described (14) two-plasmid demethylation system (Fig. 2A), seven sgRNAs were designed that targeted regions between $-1,000 \mathrm{bp}$ and the transcription start site (TSS) of the human HACE1 gene (Fig. 2B). At $48 \mathrm{~h}$ after the transfection of Hep3B and HepG2 cells with dCas9-Tet1-CD (sgRNAs 1-7) and MS2-Tet1-CD, two sgRNAs (4 and 5) were identified to increase the transcription of HACE1 mRNA by 4-5 times compared with the control group with no sgRNA (Fig. 2C). Furthermore, immunoblot analysis 

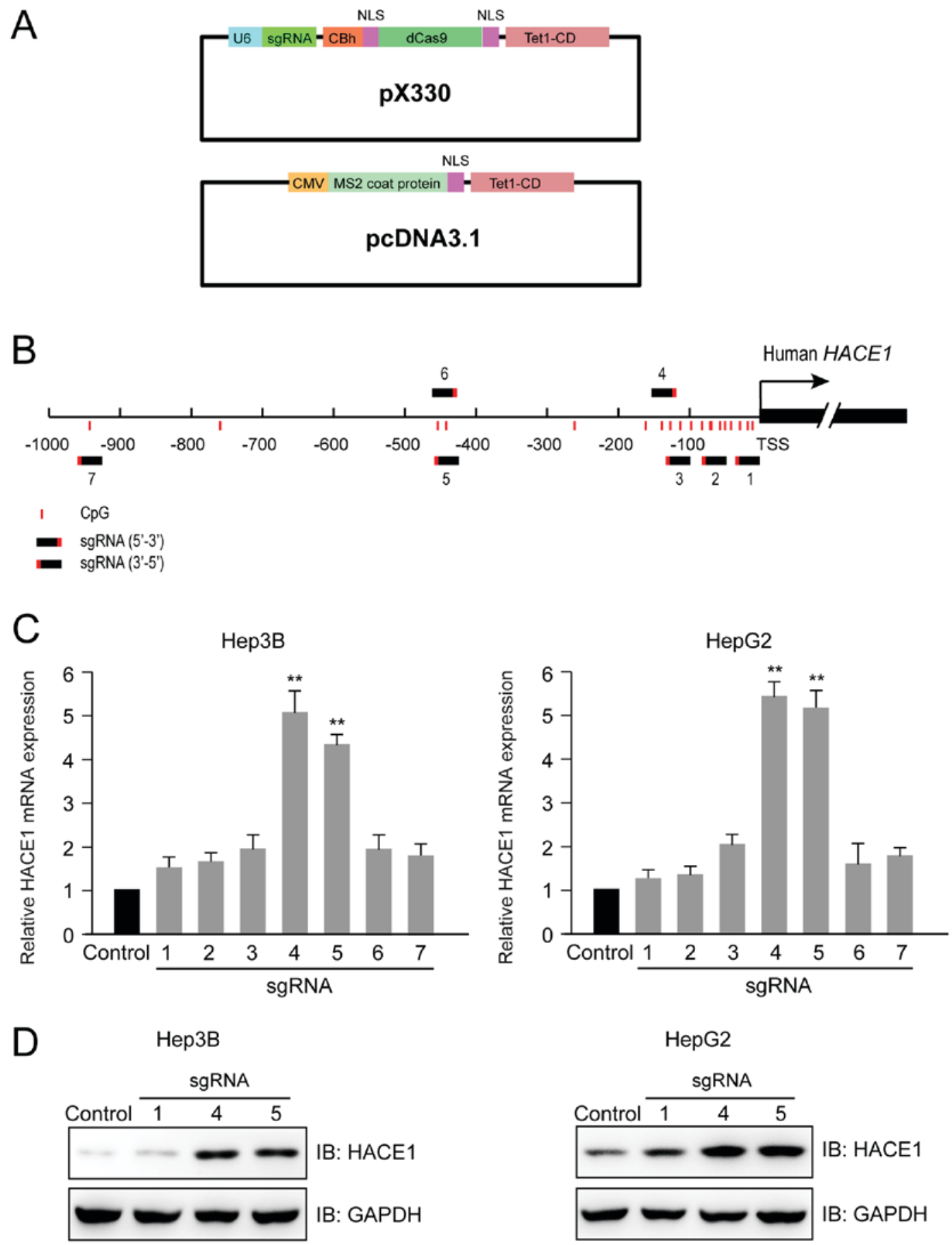

Figure 2. CRISPR-Cas-based HACE1 promoter demethylation sgRNA design and activity detection. (A) Schematic representation of the sgRNA-guided demethylation system expression cassettes. (B) Seven sgRNAs were designed to target regions between -1,000 bp and the TSS of the human HACE1 gene. sgRNAs recognizing their respective target sites were shown in a black-red colour (red colour represents the protospacer adjacent motif region), and the CpG sites were indicated with red lines. (C) HACE1 mRNA expression was detected by reverse transcription-quantitative polymerase chain reaction $48 \mathrm{~h}$ after co-transfection with sgHACE1(1-7)-guided dCas9-Tet1-CD and MS2-Tet1-CD in Hep3B and HepG2 cells. The results subsequent to normalization for the control group (without sgRNA) are presented. (D) HACE1 protein expression was detected by immunoblotting $48 \mathrm{~h}$ after co-transfection with HACE1 sgRNA (1, 4 and 5)-guided dCas9-Tet1-CD and MS2-Tet1-CD in Hep3B and HepG2 cells. Data are presented as the means \pm standard deviation of three independent experiments and were analysed using one-way analysis of variance with Tukey's post hoc test. ${ }^{* *} \mathrm{P}<0.01$ compared with control group. Cas, CRISPR-associated; CRISPR, clustered regularly interspaced short palindromic repeats; HACE1, HECT domain and ankyrin repeat containing E3 ubiquitin protein ligase 1; IB, immunoblotting; sgRNA, single guide RNA; TSS, transcription start site.

indicated that the protein levels of HACE1 were evidently increased in cells transfected with the aforementioned two $\operatorname{sgRNAs}(4$ and 5 ) compared with the control group or sgRNA 1 , which had little effect on HACE1 gene expression and was used as a negative control (Fig. 2D).
Upregulation of the target HACE1 gene by specific DNA demethylation. To determine whether the upregulated HACE1 gene transcription was a direct result of targeted demethylation that occurred at a specific HACE1 promoter sequence, the methylation status of sgRNA-targeted loci (between -500 bp and 
A

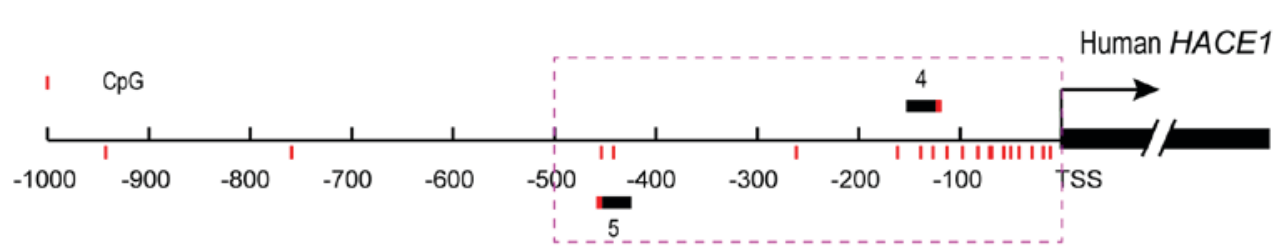

Hep3B

Control

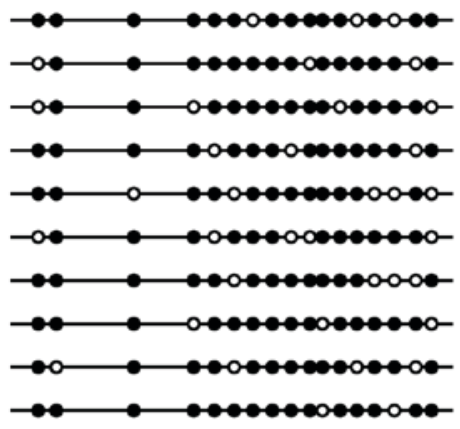

sgRNA 5

$-\infty-10-0,00000000000$

$-\infty-0-0,0000000000-$

$\rightarrow-0-0,0-00000000000$

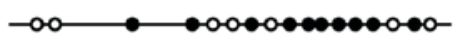

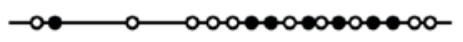

$-\infty-0-0,0000000000-$

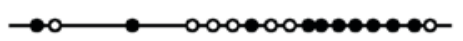

$-\infty-0-00000000000-$

-

$-\infty-0-0,00000000000-$
sgRNA 4

$\rightarrow 0-\cdots,-000000000000$

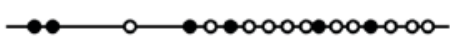

$-\infty-0,-000000000000$

$\rightarrow 0-0,000000000000$

$\rightarrow-\infty-0,00000000-00$

$\rightarrow 0-0.00000000000-$

$\rightarrow-\infty-0,-000000000-00$

$\rightarrow-0,00000000000$

$\rightarrow 00-00000000000$

$\rightarrow-\infty-\infty,-0,00000000-$

\begin{tabular}{cc}
\hline Group & Left to right: -500 to TSS site (percentages of methylated DNA) \\
\hline Control & $70 \%, 90 \%, 90 \%, 80 \%, 80 \%, 70 \%, 90 \%, 100 \%, 80 \%, 80 \%, 80 \%, 90 \%, 80 \%, 80 \%, 60 \%, 60 \%, 60 \%$ \\
sgRNA 5 & $20 \%, 30 \%, 20 \%, 20 \%, 20 \%, 20 \%, 60 \%, 50 \%, 40 \%, 90 \%, 50 \%, 80 \%, 90 \%, 90 \%, 70 \%, 60 \%, 70 \%$ \\
sgRNA 4 & $70 \%, 80 \%, 70 \%, 50 \%, 50 \%, 50 \%, 60 \%, 70 \%, 30 \%, 20 \%, 40 \%, 20 \%, 40 \%, 30 \%, 20 \%, 20 \%, 30 \%$ \\
\hline
\end{tabular}

$\mathrm{B}$

HepG2

Control

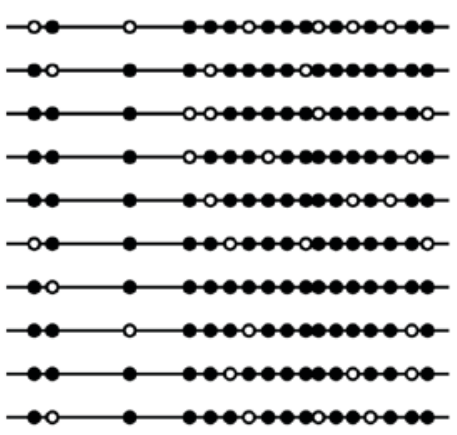

sgRNA 5

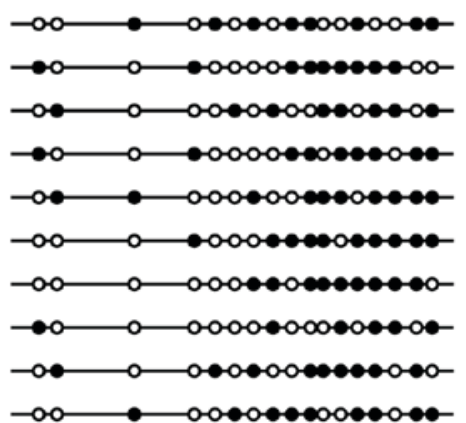

sgRNA 4

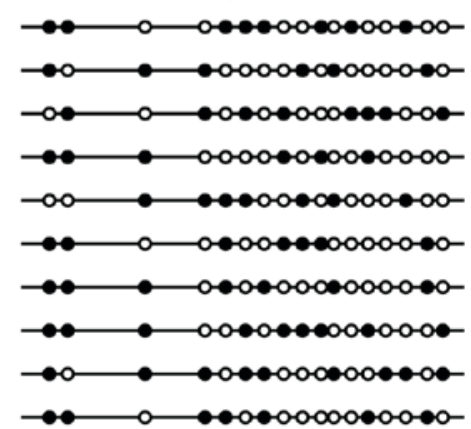

\begin{tabular}{cc}
\hline Group & Left to right: -500 to TSS site (percentages of methylated DNA) \\
\hline Control & $80 \%, 70 \%, 80 \%, 80 \%, 70 \%, 80 \%, 70 \%, 90 \%, 100 \%, 80 \%, 70 \%, 100 \%, 80 \%, 90 \%, 80 \%, 70 \%, 80 \%$ \\
sgRNA 5 & $30 \%, 30 \%, 30 \%, 30 \%, 20 \%, 20 \%, 60 \%, 50 \%, 50 \%, 80 \%, 60 \%, 70 \%, 70 \%, 90 \%, 60 \%, 70 \%, 70 \%$ \\
sgRNA 4 & $80 \%, 70 \%, 60 \%, 50 \%, 50 \%, 50 \%, 30 \%, 40 \%, 40 \%, 40 \%, 40 \%, 20 \%, 40 \%, 20 \%, 30 \%, 30 \%, 30 \%$ \\
\hline
\end{tabular}

Figure 3. CRISPR-Cas-based sgRNA system promotes HACE1 promoter demethylation. Percentages of methylated DNA at each site in the HACE1 promoter (between $-500 \mathrm{bp}$ and TSS) were determined by bisulphite sequencing $48 \mathrm{~h}$ after co-transfection with sgHACE1 (1, 4 and 5)-guided dCas9-Tet1-CD and MS2-Tet1-CD in (A) Hep3B and (B) HepG2 cells. Each line represents an individual clone, and each circle represents an individual CpG island. A black circle represents a methylated $\mathrm{CpG}$ and a white circle represents an unmethylated $\mathrm{CpG}$. The percentages of methylated $\mathrm{CpG}$ in each $\mathrm{CpG}$ island are shown. Cas, CRISPR-associated; CRISPR, clustered regularly interspaced short palindromic repeats; HACE1, HECT domain and ankyrin repeat containing E3 ubiquitin protein ligase 1; sgRNA, single guide RNA; TSS, transcription start site.

TSS) was examined using a bisulphite sequencing approach. As shown in Fig. 3A and B, expression of the two-plasmid demethylation system resulted in the removal of methyl groups from the neighbouring $\mathrm{CpG}$ islands of the HACE1 gene promoter in the Hep3B and HepG2 cell lines. Additionally, the percentages of methylated $\mathrm{CpG}$ for each $\mathrm{CpG}$ island are shown.
Demethylation of HACE1 inhibits human liver cancer cell proliferation and colony formation. A previous study reported that HACE1 has a tumour-suppressive role in hepatocellular carcinoma (5). To evaluate whether CRISPR-Cas-induced upregulation of HACE1 resulted in physiologically relevant effects, the proliferation of Hep3B and HepG2 cells was 
A

Hep3B

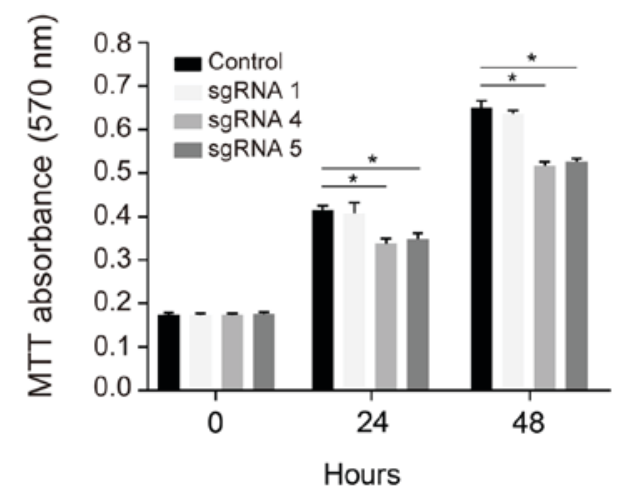

B
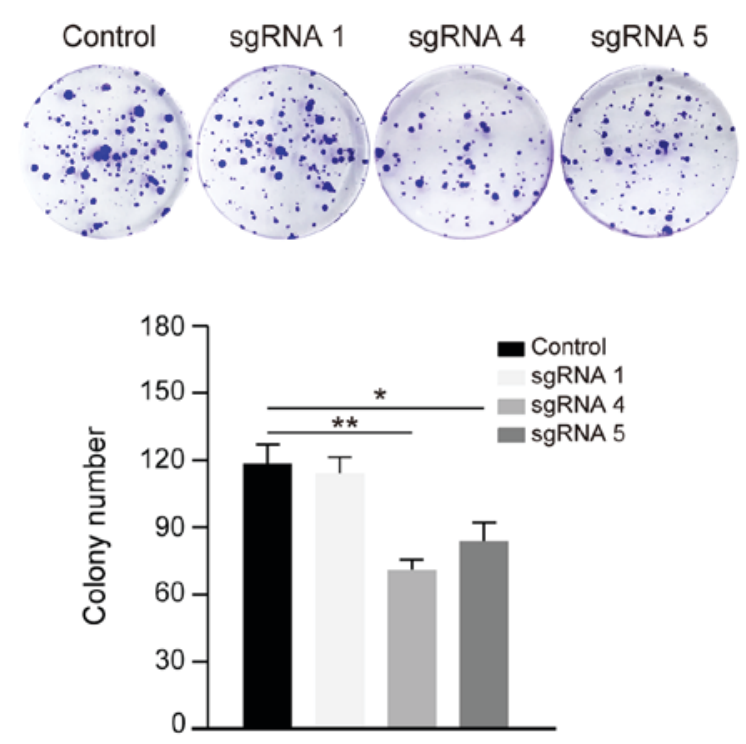

HepG2

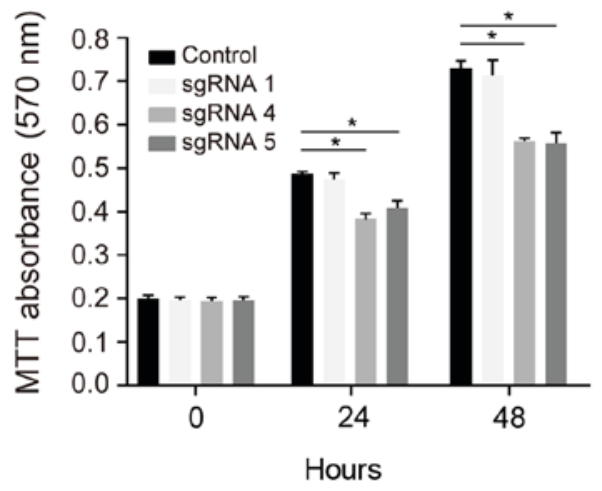

HepG2
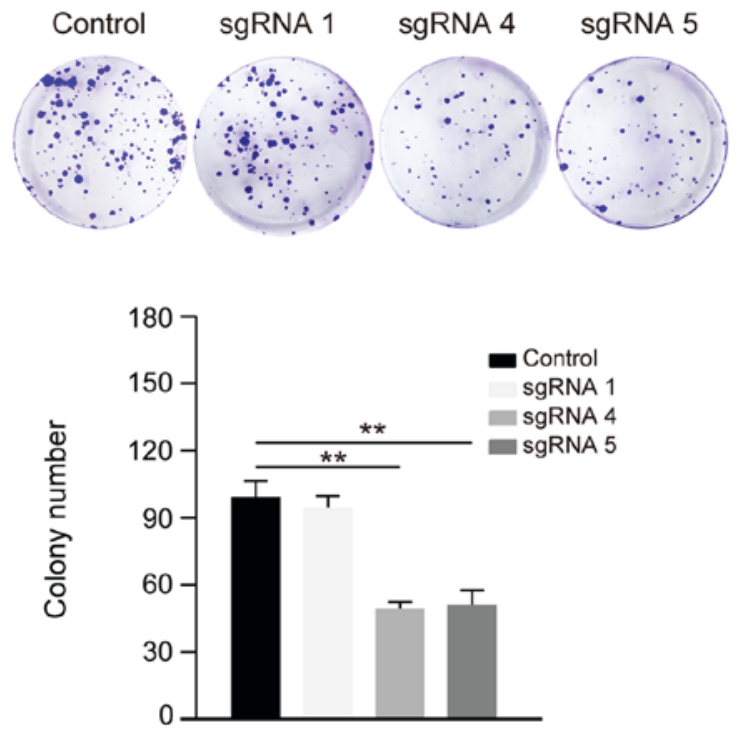

Figure 4. Demethylation of HACE1 inhibits liver cancer cell proliferation and colony formation. (A) sgRNA-mediated HACE1 demethylation inhibited liver cancer cell proliferation. Hep3B and HepG2 cells stably co-transfected with sgHACE1 (1, 4 and 5)-guided dCas9-Tet1-CD and MS2-Tet1-CD were seeded into 96-well plates, and cell proliferation was detected by an MTT assay at different time points. For this assay, $6 \mathrm{~h}$ after the cells were seeded was considered as the $0 \mathrm{~h}$ time point. Three independent experiments were performed. (B) sgRNA-mediated HACE1 demethylation inhibited liver cancer cell colony formation. Hep3B or HepG2 cells (1,000) stably co-transfected with sgHACE1 (1, 4 and 5)-guided dCas9-Tet1-CD and MS2-Tet1-CD were seeded into 6-well plates. The colonies were fixed with $4 \%$ paraformaldehyde and stained with $0.1 \%$ crystal violet 7 days after seeding. The colony numbers were counted and calculated using three samples from each group. Data are expressed as the means \pm standard deviation and were analysed using one-way analysis of variance with Tukey's post hoc test. ${ }^{*} \mathrm{P}<0.05,{ }^{* *} \mathrm{P}<0.01$. Cas, clustered regularly interspaced short palindromic repeats-associated; HACE1, HECT domain and ankyrin repeat containing E3 ubiquitin protein ligase 1; sgRNA, single guide RNA.

detected by MTT assays at 24 and $48 \mathrm{~h}$ post transfection. sgRNAs 4 and 5-induced HACE1 upregulation significantly inhibited liver cancer cell proliferation compared with the control and sgRNA 1 groups (Fig. 4A).

The demethylation systems containing sgRNAs 4 and 5 were stably expressed in Hep3B and HepG2 cells; subsequently, these cells underwent colony formation assays. Decreased colony numbers were observed in the sgRNA 4 and 5 groups compared with in the control and sgRNA 1 groups (Fig. 4B). These data suggested that CRISPR-Cas-based upregulation of HACE1 resulted in physiologically relevant effects and inhibited liver cancer cell proliferation.

Demethylation of HACE1 promotes OPTN ubiquitination and autophagy in liver cancer cells. Ubiquitination of the autophagy receptor OPTN by HACE1 has been demonstrated to activate selective autophagy, resulting in tumour suppression in lung cancer (10). In the present study, it was investigated if this phenomenon could also occur in liver cancer cells. The ubiquitination of OPTN markedly increased when cells were transfected with demethylation system vectors containing sgRNAs 4 or 5 (Fig. 5). LC3 is a central protein in the autophagy pathway, where it functions in autophagy substrate selection and autophagosome biogenesis. As a result, LC3 is the most widely used marker of autophagosomes. The ratio of LC3 II to LC3 I clearly increased when Hep3B and HepG2 cells were transfected with demethylation system vectors containing sgRNAs 4 or 5 compared with the control and sgRNA 1 groups (Fig. 5). Collectively, these results suggested that increased expression of HACE1 by targeted demethylation may promote OPTN ubiquitination and autophagy activity in liver cancer cells. 


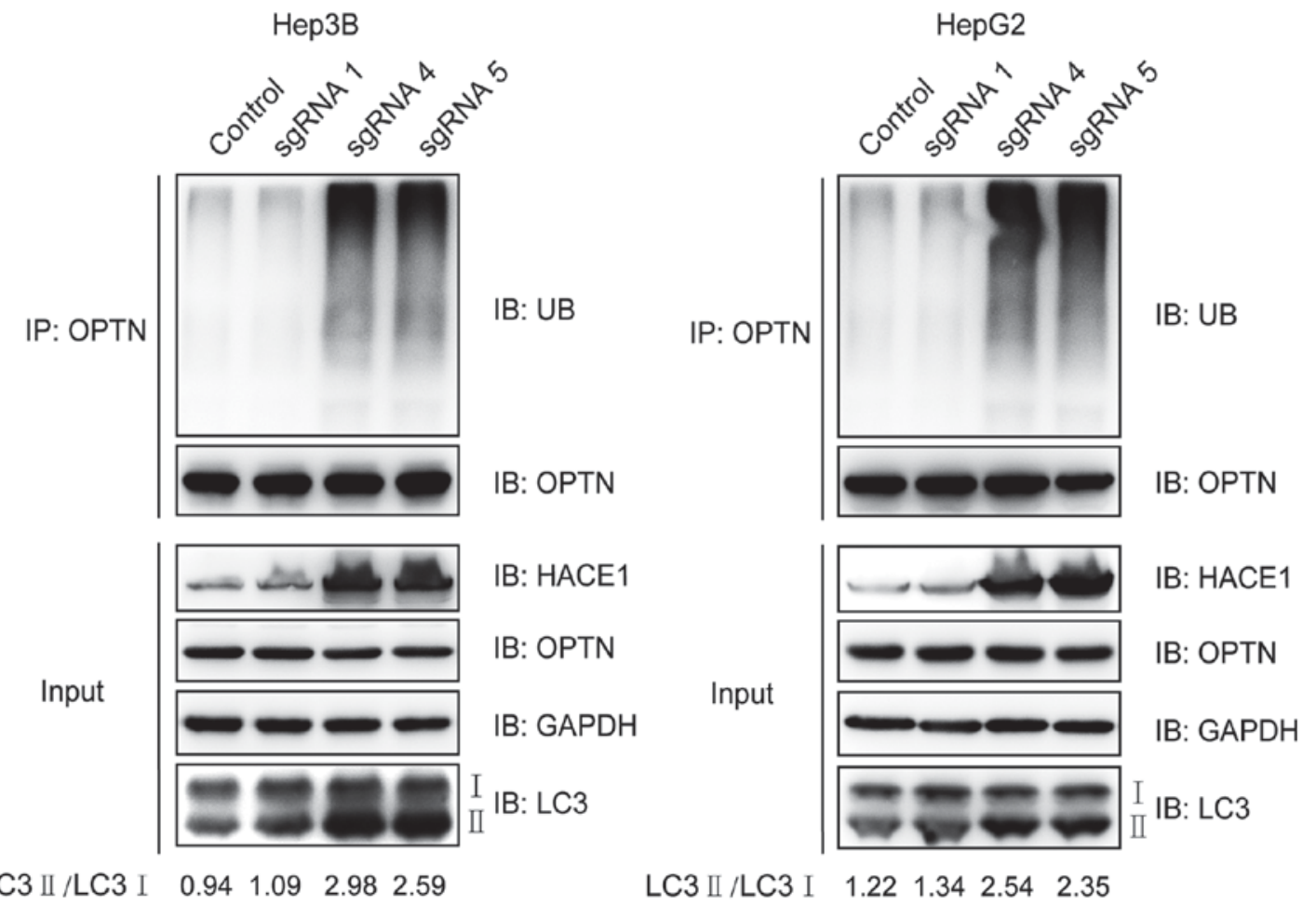

Figure 5. Demethylation of HACE1 promotes OPTN ubiquitination and autophagy. sgRNA-mediated HACE1 demethylation promoted OPTN ubiquitination. Hep3B or HepG2 cells were co-transfected with sgHACE1 (1, 4 and 5)-guided dCas9-Tet1-CD and MS2-Tet1-CD for 48 h. Subsequently, cell lysates were immunoprecipitated with an anti-OPTN antibody and subjected to immunoblotting. LC3 II/I expression was also detected. Inputs refers to whole cell lysates, and GAPDH was used as a loading control. Cas, clustered regularly interspaced short palindromic repeats-associated; HACE1, HECT domain and ankyrin repeat containing E3 ubiquitin protein ligase 1; IB, immunoblotting; LC3, microtubule-associated proteins 1A/1B light chain 3B; OPTN, optineurin; sgRNA, single guide RNA; UB, ubiquitin.

\section{Discussion}

HACE1 downregulation has been identified in numerous types of cancer, including hepatocellular carcinoma, breast cancer, colorectal cancer, gastric cancer and leukaemia $(5,11)$. Previous studies have demonstrated that decreased expression or deletion of HACE1 caused by HACE1 methylation or ubiquitination is associated with the occurrence and invasion of various types of carcinoma (18,21-23). HACE1 is a candidate tumour suppressor gene and a potential therapeutic target for several types of human cancer, including liver cancer. The results of the present study indicated that demethylation of the HACE1 promoter enhanced its expression, and inhibited proliferation and colony formation of liver cancer cells. However, a previous study demonstrated that HACE1 promotes melanoma cell migration and adhesion in vitro and that it is required for mouse lung colonization by melanoma cells in vivo (24). These findings indicated that whether HACE1 acts as a tumour suppressor gene or oncogene may depend on the cancer type.

HACE1 was first studied in Wilms' tumour and was later identified to be frequently lost or downregulated in a variety of tumours. Its role in tumour suppression has been extensively investigated, and a number of studies have indicated that HACE1 can restrain reactive oxygen species generation, control cell fate by regulating TNF receptor superfamily member $1 \mathrm{~A}$, and impede tumour growth by accelerating the ubiquitination of Rac family small GTPase $1(11,25,26)$. Notably, it has been reported that HACE1 acts as a tumour suppressor by ubiquitinating OPTN, and that it activates selective autophagy (10).
The present study indicated that demethylation of the HACE1 promoter may lead to OPTN ubiquitination and elevated protein levels of LC3 II.

A previous study indicated that the dCas9-based demethylation system has non-additive effects (14). In the present study, the two-plasmid demethylation system efficiently removed the methyl groups from neighbouring $\mathrm{CpG}$ islands on the HACE1 gene promoter in liver cancer cells.

In conclusion, further efforts are required to apply the dCas9-based demethylation system in animal models to investigate liver cancer that is aetiologically caused by HACE1 gene hypermethylation. Activating the expression of HACE1 may be a promising approach for anticancer therapy.

\section{Acknowledgements}

The authors would like to thank Professor Ronggui Hu (Institute of Biochemistry and Cell Biology, Shanghai Institutes for Biological Sciences, Chinese Academy of Sciences) for providing the demethylation system plasmids (pdCas9-Tet1-CD and pcDNA3.1-MS2-Tet1-CD) and technical support.

\section{Funding}

This study was supported by research grants from the National Natural Science Foundation Youth Fund of China (grant no. 81702622) and Liaoning Province Doctoral Startup Fund (grant no. 201501022). 


\section{Availability of data and materials}

All data generated or analysed during this study are included in the published article.

\section{Authors' contributions}

ZY and ZL conceived and designed the experiments. ZY, YL and $\mathrm{TH}$ performed the experiments, collected the data and analysed the results. ZY and ZL wrote the paper.

\section{Ethics approval and consent to participate}

Not applicable.

\section{Patient consent for publication}

Not applicable.

\section{Competing interests}

The authors declare that they have no competing interests.

\section{References}

1. Torre LA, Bray F, Siegel RL, Ferlay J, Lortet-Tieulent J and Jemal A: Global cancer statistics, 2012. CA Cancer J Clinicians 65: 87-108, 2015.

2. Siegel RL, Miller KD and Jemal A: Cancer statistics, 2018. CA Cancer J Clin 68: 7-30, 2018.

3. Waller LP, Deshpande V and Pyrsopoulos N: Hepatocellular carcinoma: A comprehensive review. World J Hepatol 7: 2648-2663, 2015.

4. Waghray A, Murali AR and Menon KN: Hepatocellular carcinoma: From diagnosis to treatment. World J Hepatol 7: 1020-1029, 2015.

5. Gao ZF, Wu YN, Bai ZT, Zhang L, Zhou Q and Li X: Tumor-suppressive role of HACE1 in hepatocellular carcinoma and its clinical significance. Oncol Rep 36: 3427-3435, 2016.

6. Villanueva A, Minguez B, Forner A, Reig M and Llovet JM: Hepatocellular carcinoma: Novel molecular approaches for diagnosis, prognosis, and therapy. Annu Rev Med 61: 317-328, 2010.

7. Anglesio MS, Evdokimova V, Melnyk N, Zhang L, Fernandez CV, Grundy PE, Leach S, Marra MA, Brooks-Wilson AR, Penninger J and Sorensen PH: Differential expression of a novel ankyrin containing E3 ubiquitin-protein ligase, Hace1, in sporadic Wilms tumor versus normal kidney. Hum Mol Genet 13: 2061-2074, 2004

8. Goka ET and Lippman ME: Loss of the E3 ubiquitin ligase HACE1 results in enhanced Rac1 signaling contributing to breast cancer progression. Oncogene 34: 5395-5405, 2015.

9. Hibi K, Sakata M, Sakuraba K, Shirahata A, Goto T, Mizukami H, Saito M, Ishibashi K, Kigawa G, Nemoto H and Sanada Y: Aberrant methylation of the HACE1 gene is frequently detected in advanced colorectal cancer. Anticancer Res 28: 1581-1584, 2008.
10. Liu Z, Chen P, Gao H, Gu Y, Yang J, Peng H, Xu X, Wang H, Yang M, Liu X, et al: Ubiquitylation of autophagy receptor optineurin by HACE1 activates selective autophagy for tumor suppression. Cancer Cell 26: 106-120, 2014.

11. Chen YL, Li DP, Jiang HY, Yang Y, Xu LL, Zhang SC and Gao H: Overexpression of HACE1 in gastric cancer inhibits tumor aggressiveness by impeding cell proliferation and migration. Cancer Med 7: 2472-2484, 2018.

12. Huang Y, de Reynies A, de Leval L, Ghazi B, Martin-Garcia N, Travert M, Bosq J, Brière J, Petit B, Thomas E, et al: Gene expression profiling identifies emerging oncogenic pathways operating in extranodal NK/T-cell lymphoma, nasal type. Blood 115: 1226-1237, 2010

13. Robertson KD: DNA methylation and human disease. Nature reviews. Genetics 6: 597-610, 2005.

14. Sakata M, Kitamura YH, Sakuraba K, Goto T, Mizukami H, Saito M, Ishibashi K, Kigawa G, Nemoto H, Sanada Y and Hibi K: Methylation of HACE1 in gastric carcinoma. Anticancer Res 29: 2231-2233, 2009.

15. Xu X, Tao Y, Gao X, Zhang L, Li X, Zou W, Ruan K, Wang F, $\mathrm{Xu}$ GL and Hu R: A CRISPR-based approach for targeted DNA demethylation. Cell Discov 2: 16009, 2016.

16. Morita S, Noguchi H, Horii T, Nakabayashi K, Kimura M, Okamura K, Sakai A, Nakashima H, Hata K, Nakashima K and Hatada I: Targeted DNA demethylation in vivo using dCas9-peptide repeat and scFv-TET1 catalytic domain fusions. Nat Biotechnol 34: 1060-1065, 2016.

17. Liu XS, Wu H, Krzisch M, Wu X, Graef J, Muffat J, Hnisz D, $\mathrm{Li} \mathrm{CH}$, Yuan B, Xu C, et al: Rescue of fragile X syndrome neurons by DNA methylation editing of the FMR1 gene. Cell 172: 979-992 e976, 2018.

18. Xu X, Li C, Gao X, Xia K, Guo H, Li Y, Hao Z, Zhang L, Gao D, $\mathrm{Xu} \mathrm{C}$, et al: Excessive UBE3A dosage impairs retinoic acid signaling and synaptic plasticity in autism spectrum disorders. Cell Res 28: 48-68, 2018.

19. Livak KJ and Schmittgen TD: Analysis of relative gene expression data using real-time quantitative PCR and the $2^{-\Delta \Delta} \mathrm{CT}$ method. Methods 25: 402-408, 2001

20. Smith-Ravin J, England J, Talbot IC and Bodmer W: Detection of c-Ki-ras mutations in faecal samples from sporadic colorectal cancer patients. Gut 36: 81-86,1995.

21. Mettouchi A and Lemichez E: Ubiquitylation of active Racl by the E3 ubiquitin-ligase HACE1. Small GTPases 3: 102-106, 2012.

22. Gacon G, Mettouchi A and Lemichez E: The tumor suppressor HACE1 targets Rac1 to ubiquitin-mediated proteasomal degradation. Med Sci (Paris) 28: 39-41, 2012.

23. Lachance V, Degrandmaison J, Marois S, Robitaille M, Génier S, Nadeau S, Angers S and Parent JL: Ubiquitylation and activation of a Rab GTPase is promoted by a beta(2)AR-HACE1 complex. J Cell Sci 127: 111-123, 2014.

24. El-Hachem N, Habel N, Naiken T, Bzioueche H, Cheli Y, Beranger GE, Jaune E, Rouaud F, Nottet N, Reinier F, et al: Uncovering and deciphering the pro-invasive role of HACE1 in melanoma cells. Cell Death Differ 25: 2010-2022, 2018.

25. Cetinbas N, Daugaard M, Mullen AR, Hajee S, Rotblat B, Lopez A, Li A, De Berardinis RJ and Sorensen PH: Loss of the tumor suppressor Hace1 leads to ROS-dependent glutamine addiction. Oncogene 34: 4005-4010, 2015.

26. Tortola L, Nitsch R, Bertrand MJM, Kogler M, Redouane Y, Kozieradzki I, Uribesalgo I, Fennell LM, Daugaard M, Klug H, et al: The tumor suppressor hacel is a critical regulator of TNFR1-mediated cell fate. Cell Rep 16: 3414, 2016. 